\title{
How Sensitive are Di-Leptons from $\rho$ Mesons to the High Baryon Density Region?
}

\author{
S.Vogel ${ }^{1}$, H. Petersen ${ }^{1,2}$, K. Schmidt ${ }^{1}$, E. Santini ${ }^{1}$, C. Sturm ${ }^{1}$ J. Aichelin ${ }^{3}$ and M. Bleicher ${ }^{1}$ \\ ${ }^{1}$ Johann Wolfgang Goethe-Universität, \\ Max-von-Laue-Straße 1, \\ 60438 Frankfurt am Main, Germany \\ ${ }^{2}$ Frankfurt Institute for Advanced Studies (FIAS), \\ Ruth-Moufang-Straße 1, \\ 60438 Frankfurt am Main, Germany and \\ ${ }^{3}$ SUBATECH, Laboratoire de Physique Subatomique et des Technologies Associées \\ University of Nantes - IN2P3/CNRS - Ecole des Mines de Nantes \\ 4 rue Alfred Kastler, F-44072 Nantes Cedex 03, France
}

\begin{abstract}
We show that the measurement of di-leptons might provide only a restricted view into the most dense stages of heavy ion reactions. Thus, possible studies of meson and baryon properties at high baryon densities, as e.g. done at GSI-HADES and envisioned for FAIR-CBM, might observe weaker effects than currently expected in certain approaches. We argue that the strong absorption of resonances in the high baryon density region of the heavy ion collision masks information from the early hot and dense phase due to a strong increase of the total decay width because of collisional broadening. To obtain additional information, we also compare the currently used approaches to extract di-leptons from transport simulations - i.e. shining, only vector mesons from final baryon resonance decays and instant emission of di-leptons and find a strong sensitivity on the method employed in particular at FAIR and SPS energies. It is shown explicitly that a restriction to $\rho$ meson (and therefore di-lepton) production only in final state baryon resonance decays provide a strong bias towards rather low baryon densities. The results presented are obtained from UrQMD v2.3 calculations using the standard set-up.

PACS numbers: 13.40.Hq,24.10.Lx,25.75.-q,25.75.Dw
\end{abstract}

Quantum-Chromo-Dynamics (QCD) predicts that the properties of hadrons change when they are brought into a (hot and/or dense) nuclear environment [1, 2]. This modification is due to the interaction with the surrounding medium which eventually leads to chiral symmetry restoration at high baryon densities and/or high temperatures [3]. The experimental verification of this theoretical prediction is one of the most challenging questions in modern strongly interacting matter physics.

Among the non-strange mesons the $\rho$ meson plays a dominant role in these investigations. It has a short lifetime and therefore it has a large probability to decay inside the reaction zone when created in heavy ion collisions. It couples strongly to nuclear resonances and, most important, it has a non-negligible chance to decay into dileptons which leave the interaction zone essentially without any further interaction. Thus, the di-lepton channel seems to offer a unique chance to study the high baryon density properties of the $\rho$ meson.

Theoretically the question of how the spectral function of the $\rho$ meson changes in the medium is still under active discussion. There is certain theoretical evidence that the $\rho$ meson is broadened if put into the nuclear medium [4, [5, 6]. In contrast, Hatsuda and Lee predicted a lowering of the $\rho$ meson mass in a nuclear environment based on QCD sum rules calculations [1]. A result which has also been found by Brown and Rho [2, 7, 8]. On the other hand, more recent calculations indicate that the pole mass of the $\rho$ meson remains almost unchanged in the nuclear medium [9, 10, 11]. However, these calculations rely on specific assumptions on the coupling strength of the $\rho$ meson to the nuclear resonances and on the branching ratios whose validity can presently only be proven by comparison to experimental data. For the present status of the theoretical spectral function calculations for vector mesons we refer to [12, 13, 14, 15, 16].

Experiments have been launched to verify these theoretical predictions. In proton-nucleus collisions [17, 18] at $12 \mathrm{GeV}$ a decrease of the $\rho$ meson mass with increasing baryon density $\rho_{B}$ as $m\left(\rho_{B}\right) / m(0)=1-0.09 \rho_{B} / \rho_{B 0}$ about half of the value predicted by theory - but without an increase of the $\rho$ meson's width has been reported. The CLAS collaboration reports that the experimental data of photon-induced reactions is compatible with no shift of the $\rho$ meson pole mass and no additional broadening to the theoretically estimated collisional broadening [19]. In contrast, the di-lepton data in In+In collisions at $158 \mathrm{AGeV}$ [20] are best described using essentially the free $\rho$ meson pole mass but a considerable broadening of the spectral function. At lower energies of $2 \mathrm{AGeV}$ (see 21] for the newest data at $1 \mathrm{GeV}$ ), the HADES collaboration has recently published di-lepton spectra [22]. Here, a deviation from the yield calculated from a hadronic cocktail fit in the vicinity of the $\rho$ meson mass is visible 
but due to the many sources of di-leptons there is no conclusive explanation for that suppression yet. How much of these different experimental findings can be exclusively attributed to the different environments, i.e. cold nuclear matter in proton-nucleus reactions, an expanding meson dominated fireball after a possible phase transition from a quark gluon plasma in high energy nucleus-nucleus collisions at the SPS or a baryon dominated expansion in reactions at about $2 \mathrm{AGeV}$ is still a matter of debate.

To link the final state di-lepton data to the in-medium spectral functions of the hadrons detailed quantitative theoretical simulations of the baryon density distribution at the $\rho$ meson production and decay/absorption point are necessary. This allows then to calculate di-lepton spectra from the simulations. Unfortunately, up to now different approaches are used to convert the calculated hadron spectra into di-leptons. In general four different approaches can be identified:

- Explicit propagation of stable particles, baryon and meson resonances, decays of resonances into other mesons (especially baryonic resonances into $\rho$ mesons), as well as $\pi \pi \rightarrow \rho$ scattering. Di-leptons are emitted continuously from vector mesons and baryon resonances with their respective locally given total width while the resonances are propagated ("shining") [23, 24, 25, 26, 27],

- Explicit propagation of stable particles, baryon and meson resonances, decay of resonances into other mesons (especially baryonic resonances into $\rho$ mesons), as well as $\pi \pi \rightarrow \rho$ scattering. Di-leptons are only emitted at the point of decay (not absorption) of the hadronic resonance [28],

- Explicit propagation of nucleons, pions and nucleon resonances while vector meson degrees of freedom are not propagated explicitly. Di-leptons are produced via the eVMD model from nucleonic resonances at the point of the decay of the nucleonic resonances. This model assumes di-lepton production via intermediate $\rho / \omega$ states [16, 29],

- Explicit propagation of nucleons, pions, kaons and $\Delta$ resonance. Di-leptons are produced by decay of nucleonic resonances according to the branching ratios. This is supplemented by the di-lepton production from mesonic resonances, which are calculated by folding the $\sqrt{s}$ distribution of the elementary nucleon-nucleon collisions with the mesonic production cross sections and the corresponding branching ratios into di-leptons [30].

While the first method is sensitive to all stages of the collision and allows for a dynamical treatment of the collisional broadening, the other methods rely on the actual decay of the meson or baryon resonance. Thus, the different approaches probe different baryon density regimes and might therefore provide different results for the extracted di-lepton rates. Another problem is posed by

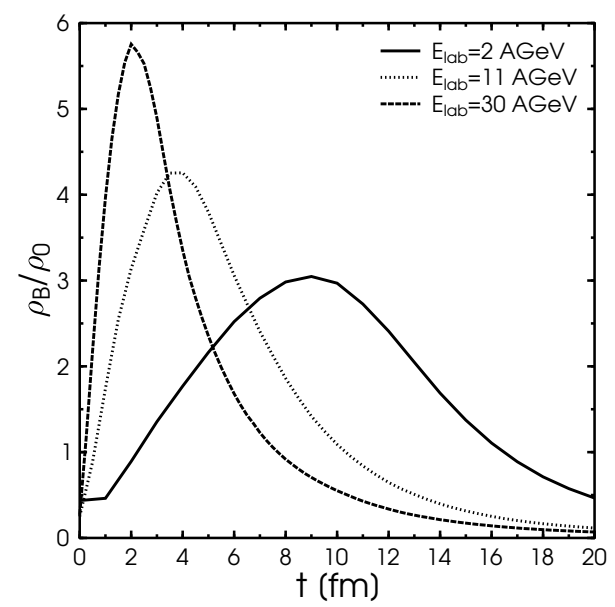

FIG. 1: (Color online) Time evolution of the local rest frame baryon density $\rho_{B}$ averaged over the positions of the individual hadrons for central $\mathrm{Au}+\mathrm{Au} / \mathrm{Pb}+\mathrm{Pb}$ reactions at various beam energies.

the implementation of bremsstrahlung especially at low beam energies. This discussion has recently been revived by the calculations of [27, 30, 31, 32].

In this situation it is necessary to study the general differences between the above discussed approaches and explore the baryon density probed by the $\rho$ meson in the FAIR energy regime. This helps to provide a theoretical error margin for further detailed model studies on the change of the in-medium spectral functions at these energies.

We perform this study for massive nuclear reactions in the energy range of $2 A \mathrm{GeV} \leq E_{\text {lab }} \leq 30 A \mathrm{GeV}$. This range marks the expected transition towards chiral symmetry restoration, but also the transition from baryon dominated to meson dominated matter. Dedicated facilities to explore this energy domain are the FAIR project at GSI [33] and the critRHIC program at BNL [34]. For our studies we employ the UrQMD(v2.3) model [35, 36, 37]. It is a non-equilibrium transport approach based on the covariant propagation of hadrons and strings. All elementary cross sections are calculated by the principle of detailed balance, from the additive quark model or are derived from available data. This model allows to study the full space time evolution for all hadrons, resonances and their decay products. It permits to explore the emission patterns of the resonances in detail and to gain insight into the origin of the resonances $39,40,41,42,43]$.

In this approach the resonances are treated with their vacuum properties during the whole evolution of the reaction. However, one should note that the particle properties are dynamically modified in a hot and/or dense medium due to the coupling of the $\rho$ meson to the surrounding hadrons (at SIS energies especially the baryon resonances are important [43]). The $\rho$ meson is assumed 


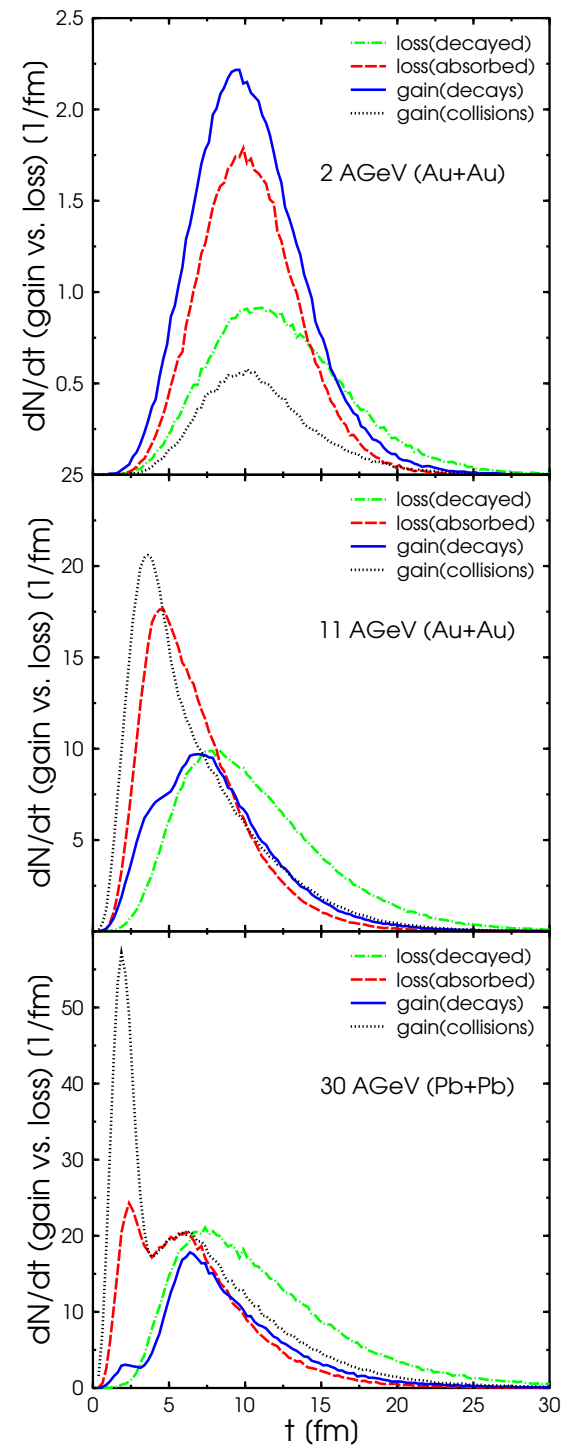

FIG. 2: (Color online) Gain and loss rates of $\rho$ mesons separated gain from collisions ("gain(collisions)"), gain from decay ("gain(decays)") and loss due to absorption ("loss(absorbed)") and loss in decays ("loss(decayed)"). From top to bottom we display central $\mathrm{Au}+\mathrm{Au} / \mathrm{Pb}+\mathrm{Pb}$ collisions at 2,11 and $30 \mathrm{AGeV}$.

to have a lifetime according to an exponential distribution with a mean lifetime $\tau$ of $1 / \Gamma_{\text {pole }} \simeq 1 / 150 \mathrm{MeV}$, in addition collisional broadening is implicitly taken into account for the calculation of di-leptons by the interaction of the $\rho$ meson with the evolving medium.

UrQMD has been successfully applied to study light and heavy ion reactions from $E_{\text {beam }}=90 \mathrm{~A} \mathrm{MeV} \mathrm{to}$ $\sqrt{s_{N N}}=200 \mathrm{GeV}$. The present version of the transport model is able to describe the yields and the transverse momentum spectra of different particles in protonproton, proton-nucleus and nucleus-nucleus collisions 37, 44. Detailed comparisons of UrQMD with a large body of experimental data at SIS and FAIR energies can

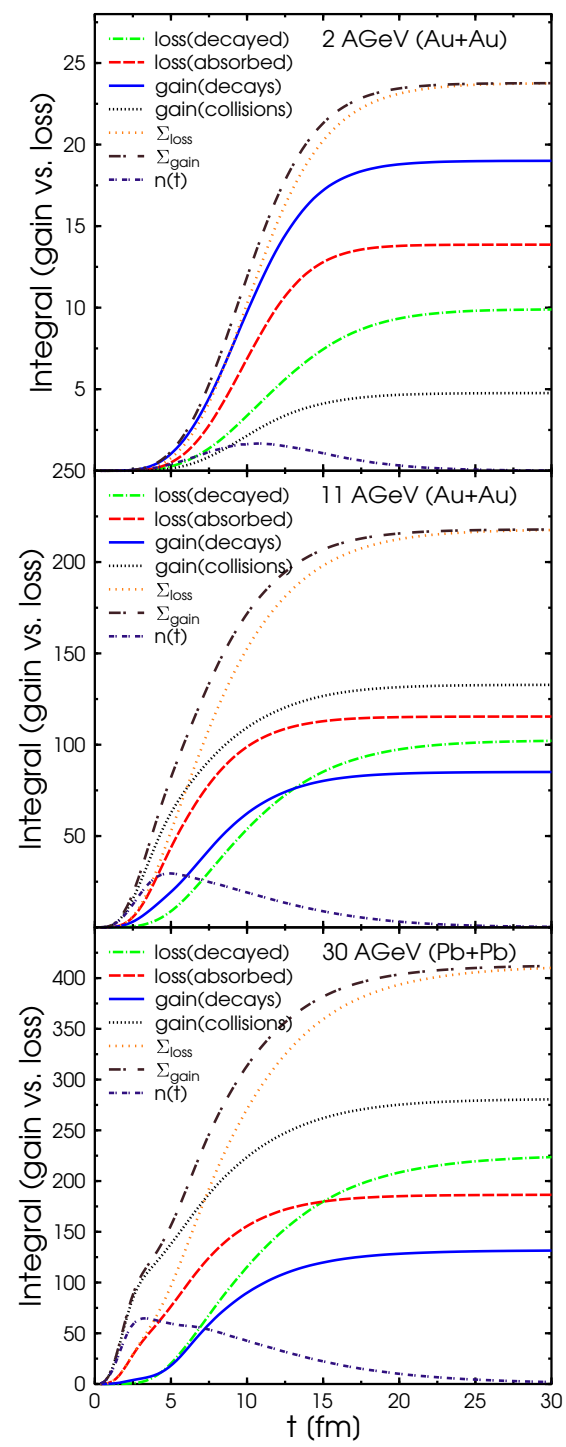

FIG. 3: (Color online) Gain and loss of $\rho$ mesons separated for collisions and decay. From top to bottom we display central $\mathrm{Au}+\mathrm{Au} / \mathrm{Pb}+\mathrm{Pb}$ collisions at 2,11 and $30 \mathrm{AGeV}$. We show as well the difference of gain and loss, the number of $\rho$ mesons present in the system as a function of time. From top to bottom we display central $\mathrm{Au}+\mathrm{Au} / \mathrm{Pb}+\mathrm{Pb}$ collisions at 2, 11 and $30 \mathrm{AGeV}$.

be found in 37, 45]. For further details of the model the reader is referred to [35, 36, 37].

Let us start the discussion by displaying the time evolution of the baryon density in central $\mathrm{Au}+\mathrm{Au} / \mathrm{Pb}+\mathrm{Pb}$ reactions at 2, 11 and $30 \mathrm{~A} \mathrm{GeV} \mathrm{(see} \mathrm{Fig.} \mathrm{1).} \mathrm{The} \mathrm{baryon}$ density is averaged over all hadron positions and is calculated locally in the rest frame of the baryon current (Eckart frame) averaged over the position of every baryon as $\rho_{B}=j^{0}$ with $j^{\mu}=\left(\rho_{B}, \overrightarrow{0}\right)$. Details on the calculation of the baryon density are discussed in the Appendix. Note that the maximal baryon density grows with increasing beam energy. The question to be asked is, how 




FIG. 4: (Color online) Baryon density distribution at the space points where the $\rho$ mesons decay. From top to bottom we display central $\mathrm{Au}+\mathrm{Au} / \mathrm{Pb}+\mathrm{Pb}$ collisions at 2, 11 and 30 $\mathrm{AGeV}$

sensitive are di-lepton observables on the high baryon density stage of the collisions?

In order to investigate this question, we first review the different production and loss mechanisms for the $\rho$ meson. In UrQMD the $\rho$ meson can be produced from the decay of a high mass (meson or baryon) resonance or directly in a collision of two particles (e.g. $\pi+\pi \rightarrow \rho$ ) which includes also the production from string fragmentation. The $\rho$ meson is destroyed by two different mechanisms. It can decay ("loss (decayed)", e.g. $\rho \rightarrow \pi^{+} \pi^{-}$) or it can be absorbed in collisions ("loss (absorbed)", e.g. $\left.\rho+\pi \rightarrow a_{1}\right)$.

In Fig. 2 we show the respective gain and loss rates of $\rho$ mesons separated for the different contributions for central $\mathrm{Au}+\mathrm{Au}$ and $\mathrm{Pb}+\mathrm{Pb}$ reactions at $E_{\text {lab }}=2,11$

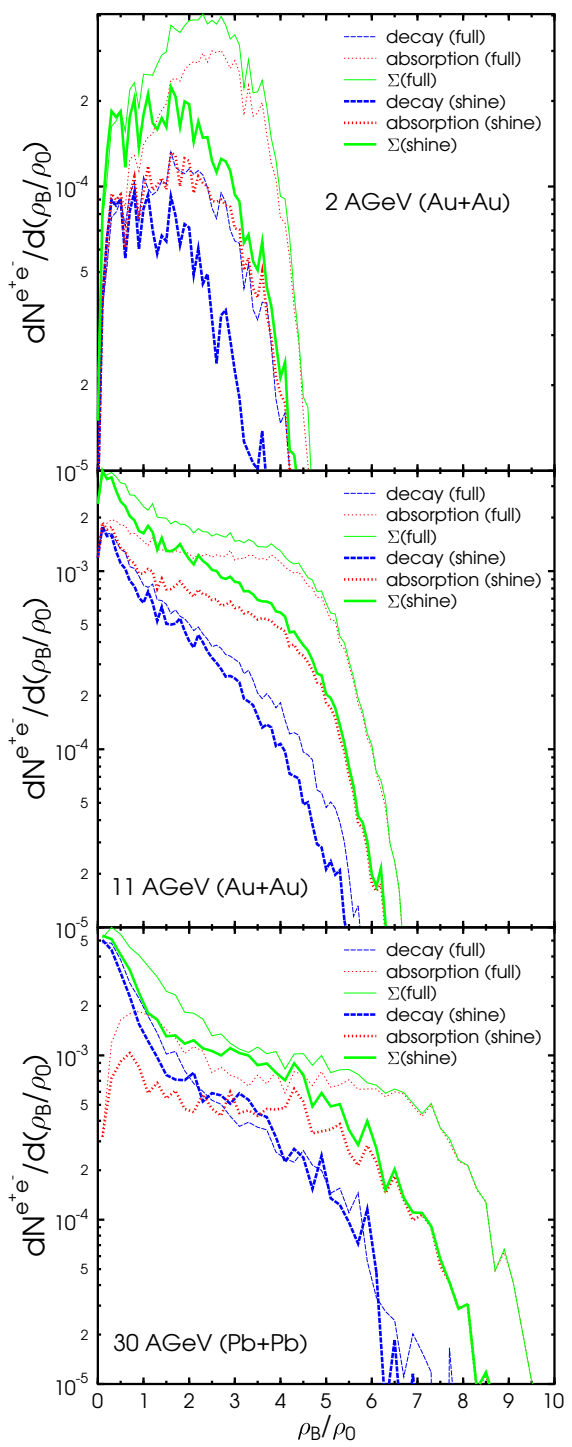

FIG. 5: (Color online) Distribution of the baryon density at which the $e^{+} e^{-}$-pairs from the $\rho$ vector meson are emitted. The thin lines denote calculation where the full branching ratio into di-leptons is attached to both the $\rho$ decay and the $\rho$ absorption vertices. The thick lines indicate the results obtained by the shining method with continuous emission of di-leptons. From top to bottom we display central $\mathrm{Au}+\mathrm{Au} / \mathrm{Pb}+\mathrm{Pb}$ collisions at 2, 11 and $30 \mathrm{AGeV}$.

and $30 \mathrm{~A} \mathrm{GeV}$. The different processes [46] in which a $\rho$ meson is produced are denoted as "gain (collisions)" or "gain (decays)" (meaning the stem from the decay of another resonance). The loss term differentiates also between $\rho$ mesons which have decayed "loss (decayed)" and those which are absorbed in collisions "loss (absorbed)" as discussed above.

At $2 A \mathrm{GeV}$, one observes that the production rate of $\rho$ mesons is dominated by the decay of resonances $(\sim 80 \%$, full line) as compared to the formation in a s-channel $\pi \pi$ scattering ( $\sim 20 \%$, dotted line). This observation is in 
line with the expectations that a dominant production channel for the $\rho$ meson in low and intermediate energy heavy ion collisions is the decay of baryon resonances [38]. A previous detailed analysis of the $\rho$ meson production channels at $2 \mathrm{~A} \mathrm{GeV}$ within the UrQMD approach, found in [43] confirms this interpretation in detail. The maximum of the $\rho$ meson production rate coincides with the maximum baryon density around $t \sim 9 \mathrm{fm}$. However, these $\rho$ mesons are subject to frequent interactions with the surrounding baryons resulting in rather short life times of the $\rho$ mesons as indicated by the large absorption rate (dashed line). Only towards the end of the high density stage (when the $\rho$ absorption processes, e.g. $\rho+B \rightarrow B^{* \prime}$ cease) $\rho$ mesons can decay directly as denoted by the dashed-dotted line. In the present model $\rho$ meson absorption accounts for the main loss of $\rho$ mesons, while the decay accounts for only $30 \%$ of the $\rho$ meson loss. It is clear that these features might lead to different time-dependent di-lepton yields, depending on the method with which the di-lepton rates are extracted from the numerical simulation.

At higher energies $(11 A \mathrm{GeV}, 30 A \mathrm{GeV})$ this low energy line of arguments changes. Here one observes two distinct phases for the production and decay/absorption of the $\rho$ meson. Initially $\rho$ meson production from collisions proceeding either via string formation and fragmentation or via meson-meson scattering dominates the gain term (dotted line). However, also the absorption probability is rather high in this stage of the reaction resulting in a quick re-absorption of the $\rho$ meson (dashed line). For di-lepton calculations it becomes clear that only a "shining" approach has the potential to provide information on this stage, whereas the approaches which depends on the decay of the resonance do not allow to extract this information. However, also at higher energies, the production of $\rho$ mesons from resonance decays in the late stage of the reaction is sizeable. This is evident because the sequential processes $N N \rightarrow B^{*}+X, B^{*} \rightarrow \rho+X$ will need a certain time and therefore triggers on later stages of the reaction. Thus, the $\rho$ meson production from baryon resonance decays again leads towards a selftriggering of $\rho$ meson production and subsequent decay at rather moderate densities, even at energies of $11 A \mathrm{GeV}$ and $30 A \mathrm{GeV}$.

To gauge our assumptions for the absolute importance of the different processes the integrated $\rho$ meson rates are shown in Fig. 3. The nomenclature is the same that has been used in Fig. 2. The temporal evolution of the integrals for three values of beam energies from $E_{\text {lab }}=$ $2 A \mathrm{GeV}$ to $E_{\text {lab }}=30 A \mathrm{GeV}$ is calculated. In addition, the sum of the gain $\left(\Sigma_{\text {gain }}\right)$ and the sum of the loss terms $\left(\Sigma_{\text {loss }}\right)$ are displayed. If one subtracts the sum of the loss terms from the sum of the gain terms one gets the yield $(n(t))$ of $\rho$ mesons which is present at each time step of the collision.

As discussed above, at the lowest energy the gain via resonance decays dominates over the gain from direct $\rho$ meson production due to kinematical constraints. How-

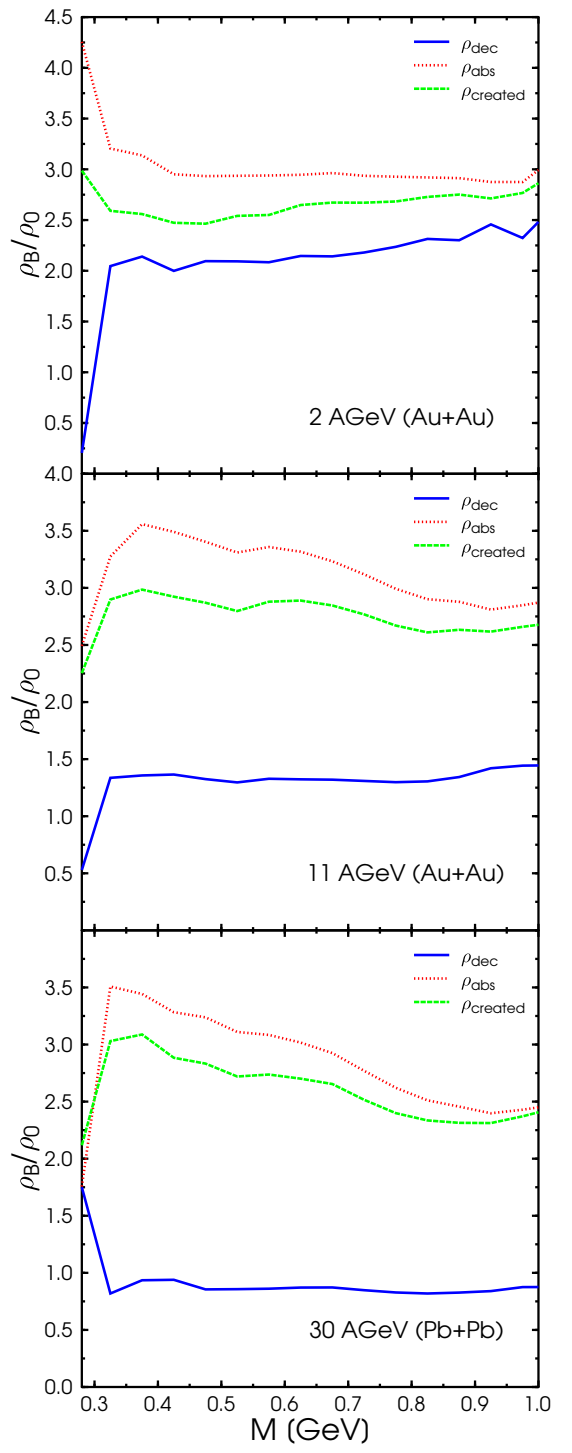

FIG. 6: (Color online) Average baryon density experienced by a $\rho$ meson as a function of the mass of the $\rho$ meson. The results of the present calculation for the creation, absorption and decay point of the respective $\rho$ meson are shown. From top to bottom we display central $\mathrm{Au}+\mathrm{Au} / \mathrm{Pb}+\mathrm{Pb}$ collisions at 2, 11 and $30 \mathrm{AGeV}$.

ever, this behaviour reverses already at $E_{\text {lab }}=11 A \mathrm{GeV}$. At the highest energy $\left(E_{\text {lab }}=30 A \mathrm{GeV}\right)$ displayed already a factor of 2.5 more $\rho$ mesons are produced in collisions than in decays of other resonances. For the loss term we observe a dominance of the absolute value of decayed $\rho$ mesons at $E_{\text {lab }}=30 \mathrm{~A} \mathrm{GeV}$.

Let us investigate the baryon density distribution at the space-time position of the $\rho$ meson decay and absorption in more detail. Fig. 4 shows the probability distribution of the baryon density at the instant of the $\rho$ decay (dashed line), $\rho$ absorption (dotted line) and for the sum of both (full line).

At $2 A \mathrm{GeV}$ (top figure) one clearly observes that 
absorption of the $\rho$ meson in the most dense medium reached at SIS energies $\left(\sim 3 \rho_{B} / \rho_{B 0}\right)$ is already a strong effect. If only decaying $\rho$ mesons are taken into account the effective density probed reduces to $\left(\sim 2 \rho_{B} / \rho_{B 0}\right)$ At higher beam energies $(11 A \mathrm{GeV}, 30 A \mathrm{GeV})$ this splitting in the density between decaying $\rho$ mesons and absorbed $\rho$ mesons becomes even more pronounced. Here, absorption processes are strongly dominating the high baryon density stage, while decays populate the low baryon density region.

To relate the present discussion directly to the sensitivity of di-leptons to the most dense stages of the reaction, di-lepton rates are calculated as a function of the baryon density at which the di-leptons are emitted. The di-lepton calculations are based on standard cross sections as e.g. given in 28]. Fig. 5 gives the distribution of the baryon density at which the $e^{+} e^{-}$-pairs from the $\rho$ vector meson are emitted (from top to bottom central $\mathrm{Au}+\mathrm{Au} / \mathrm{Pb}+\mathrm{Pb}$ collisions at 2, 11 and $30 A \mathrm{GeV}$ are shown). For each energy, the di-lepton production as a function of baryon density is separated into a part where the initial $\rho$ meson decays (dashed lines) and a part where the $\rho$ meson was absorbed by the medium (dotted lines), the sum of both contributions provides the total emission rate of di-leptons and is shown as full lines.

Two different scenarios related to the initially discussed theoretical approaches for the di-lepton extraction can be discussed:

1. In the first scenario, the full branching ratio into di-leptons is attached to both the $\rho$ decay and the $\rho$ absorption vertices (shown as thin lines). This provides the most optimistic reach towards high baryon densities, as it assumes that no collisional broadening takes place even in the most dense stage of the reaction (i.e. its a full vacuum baseline calculation). On the one hand, this setting is similar to the one employed in models that calculate dileptons from folding the $\sqrt{s}$ distribution of nucleonnucleon collisions as it assumes no interaction of the produced $\rho$ meson with the medium, i.e. absorption. On the other hand, if one omits the di-leptons emitted in the absorption process, these calculations are similar to previous calculations that assume di-lepton production only from the late stage decays of (baryon) resonances.

2. This scenario is set in contrast to the shining approach (indicated by thick lines). In the shining method, a continuous emission of di-leptons is assumed over the whole lifetime of the $\rho$ meson (generally for all relevant hadrons). The di-lepton emission rate is then integrated over time, taking the collisional broadening for each individual vector meson in its surrounding into account. Due to the strong collisional broadening in the medium, a drastic reduction in the analysis reach of di-leptons towards high densities (shown in Fig. 5 from the comparison of the thick and thin full lines) results.
At SIS and FAIR energies, the effective baryon density probed by $\rho$ mesons decaying into di-leptons is reduced to 1-2 $\rho_{B} / \rho_{B 0}$ in contrast to the expected values around $2-3 \rho_{B} / \rho_{B 0}$ from scenario 1. At higher energies $(11 A \mathrm{GeV}, 30 A \mathrm{GeV})$, the reach of di-leptons into the most dense stage is also strongly reduced. In addition, late stage decays of baryon resonances and $\rho$ mesons provide a strong trigger towards low baryon densities, resulting in strong peak of the di-lepton emission rate below 1-1.5 $\rho_{B} / \rho_{B 0}$. This low density peak might possibly blur the view on the most interesting di-leptons from the most dense stages of the reaction.

Finally, we explore the average baryon density experienced by $\rho$ mesons with different masses. This is important to understand whether any prominent features are present in the $\rho$ meson mass region between $400-600$ $\mathrm{MeV}$, that is important for the intermediate mass dilepton enhancement. Fig. 6 depicts the average baryon density experienced by a $\rho$ meson as a function of the mass of the $\rho$ meson. Fig. 6 gives the results of the present calculation for the creation, absorption and decay point of the respective $\rho$ meson. One observes that the baryon density of the system is constant as a function of the mass, indicating that most $\rho$ mesons, independent of their mass decay at a certain (low) baryon density, as argued before. Note that the baryon density where $\rho$ mesons are absorbed is higher, which is in line with the previous discussion.

In conclusion, we have shown that the measured dileptons provide only a restricted view into the most dense stages of the reaction despite the fact that electromagnetic probes leave the reaction zone without any further interaction. Thus, possible studies of meson and baryon properties at highest baryon densities might be blurred. For the $\rho$ meson we have shown that the baryon density probed in the di-lepton decay channel does depend on the method of di-lepton extraction employed. We argued that the absorption of resonances in the high baryon density region of a heavy ion collision masks information from the early hot and dense stage. To demonstrate this, we have split the contributions of the loss term of the $\rho$ meson yield into "loss(absorbed)" and "loss(decayed)" and have shown that at early times, i.e. at the highest baryon densities the absorption results in substantial reduction of the $\rho$ meson life times. The explored energy regime of low and intermediate energies will be investigated with the planned critRHIC program and is in the focus of the future FAIR project.

\section{Acknowledgments}

We thank D. Cozma and E. Bratkovskaya for useful comments. The computational resources have been provided by the Center for the Scientific Computing (CSC) at Frankfurt. This work was supported by GSI, DAAD (PROCOPE) and BMBF. H.P. thanks the Deutsche 
Telekom Stiftung for financial support. H.P. and S.V. thank the Helmholtz association for additional financial support.

\section{Appendix: The four-current method}

The local baryon density at a space point $i$ is the zeroth component of the baryon four-current $j^{\mu}=\left(\rho_{B}, \vec{j}\right)$. The local rest frame $(\mathrm{RF})$ baryon density at this space point is defined in the frame where the three-current vanishes, $j_{R F}^{\mu}=\left(\rho_{B, R F}, \vec{j}_{R F}\right)$, with $\vec{j}_{R F}=0$. This definition is known as the Eckart frame. Other definitions are possible, e.g. in the Landau frame, the energy-momentum tensor is at rest while a baryon three-current might still be present. We believe however, that the Eckart frame definition captures the relevant physics at the energy regime under investigation.

In the context of the UrQMD model quantities are (per default) calculated in the computational frame (CF) which is (for symmetric systems) the center-of-mass frame of the whole heavy ion collision. In the computational frame one is only able to evaluate $j_{C F}^{\mu}=$ $\left(\rho_{B, C F}, \vec{j}_{C F}\right)$ where $\rho_{B, C F}=N / V$ is the baryon density ( $N$ denoting baryon number in the volume, $V$ being the small local volume around the position $i$ ) and $\vec{j}_{C F}=\rho_{B, C F} \vec{\beta}$. In the limit of an infinitely small volume, the density $\rho_{B, C F}$ is a sum of Gaussians at position $i$ :

$$
\begin{gathered}
\rho_{C F}\left(\overrightarrow{r_{i}}\right)=\sum_{j=1}^{N}\left(\frac{1}{\sqrt{2 \pi} \sigma}\right)^{3} \gamma_{z} \mathrm{e}^{\left(-\frac{\left(x-x_{0}\right)^{2}+\left(y-y_{0}\right)^{2}+\left(z-z_{0}\right)^{2} \gamma_{z}^{2}}{2 \sigma^{2}}\right)} \\
=\sum_{j=1}^{N} P_{j}
\end{gathered}
$$

i.e., a three-dimensional in $z$-direction contracted and normalised Gaussian with $\gamma_{z}=1 / \sqrt{1-\beta_{z}^{2}}$ being the Lorentz factor for the particle under consideration. The normalisation is different for individual particles due to the different $\gamma$ factors. The nominal width of the Gaussian is case $\sigma=1.5 \mathrm{fm}$. The particle that defines position $i$ has to be included in the sum because one is interested in the baryon density in the local rest frame of the cell and not in the density around a particle in its rest frame.

The velocity of the cell is computed with the same Gaussians as used for the density calculation as weighting functions. Therefore, the velocity of the cell in the computational frame is:

$$
\vec{\beta}_{C F}=\frac{\sum_{j=1}^{N}\left(\frac{\overrightarrow{p_{j}}}{E_{j}}\right) \cdot P_{j}}{\sum_{j=1}^{N} P_{j}}
$$

The last step is to perform a general Lorentz boost of the four-vector $j_{C F}^{\mu}$ into the local rest frame of the cell. I.e. a Lorentz transformation with the velocity of the cell $\overrightarrow{\beta_{C F}}$. The transformation matrix is the following:

$$
\left(\begin{array}{cccc}
\gamma & -\beta_{x} \gamma & -\beta_{y} \gamma & -\beta_{z} \gamma \\
-\beta_{x} \gamma & 1+(\gamma-1) \frac{\beta_{x}^{2}}{\beta^{2}} & (\gamma-1) \frac{\beta_{x} \beta_{y}}{\beta^{2}} & (\gamma-1) \frac{\beta_{x} \beta_{z}}{\beta^{2}} \\
-\beta_{y} \gamma & (\gamma-1) \frac{\beta_{y} \beta_{x}}{\beta^{2}} & 1+(\gamma-1) \frac{\beta_{y}^{2}}{\beta^{2}} & (\gamma-1) \frac{\beta_{y} \beta_{z}}{\beta^{2}} \\
-\beta_{z} \gamma & (\gamma-1) \frac{\beta_{z} \beta_{x}}{\beta^{2}} & (\gamma-1) \frac{\beta_{z} \beta_{y}}{\beta^{2}} & 1+(\gamma-1) \frac{\beta_{z}^{2}}{\beta^{2}}
\end{array}\right)
$$

with $\beta^{2}=\beta_{x}^{2}+\beta_{y}^{2}+\beta_{z}^{2}$ and $\gamma=1 / \sqrt{1-\beta^{2}}$. The zerocomponent of the transformed $j^{\mu}$ four-vector is the local rest frame baryon density we are interested in.
[1] T. Hatsuda and S. H. Lee, Phys. Rev. C 46, 34 (1992).

[2] G. E. Brown and M. Rho, Phys. Rev. Lett. 66, 2720 (1991).

[3] R. Rapp and J. Wambach, Adv. Nucl. Phys. 25, 1 (2000) arXiv:hep-ph/9909229.

[4] R. D. Pisarski, Phys. Rev. D 52, 3773 (1995) arXiv:hep-ph/9503328.

[5] R. Rapp, G. Chanfray and J. Wambach, Phys. Rev. Lett. 76, 368 (1996) arXiv:hep-ph/9508353.

[6] R. Rapp, G. Chanfray and J. Wambach, Nucl. Phys. A 617, 472 (1997) arXiv:hep-ph/9702210.

[7] G. Q. Li, C. M. Ko and G. E. Brown, Phys. Rev. Lett. 75, 4007 (1995) arXiv:nucl-th/9504025.

[8] G. E. Brown and M. Rho, Phys. Rept. 363, 85 (2002) arXiv:hep-ph/0103102.

[9] H. van Hees and R. Rapp, Phys. Rev. Lett. 97, 102301 (2006) arXiv:hep-ph/0603084.

[10] J. Ruppert and T. Renk, Eur. Phys. J. C 49, 219 (2007) arXiv:nucl-th/0609083.

[11] K. Dusling, D. Teaney and I. Zahed, Phys. Rev. C 75, 024908 (2007) arXiv:nucl-th/0604071.

[12] M. Post and U. Mosel, Nucl. Phys. A 699, 169 (2002)
arXiv:nucl-th/0108017.

[13] P. Muehlich, V. Shklyar, S. Leupold, U. Mosel and M. Post, Nucl. Phys. A 780, 187 (2006) arXiv:nucl-th/0607061.

[14] K. Gallmeister, T. Leitner, S. Leupold, U. Mosel, P. Muehlich, L. Alvarez-Ruso and V. Shklyar, Nucl. Phys. A 782, 166 (2007) arXiv:nucl-th/0608025.

[15] R. Rapp, Nucl. Phys. A 782, 275 (2007) arXiv:nucl-th/0608022.

[16] E. Santini, M. D. Cozma, A. Faessler, C. Fuchs, M. I. Krivoruchenko and B. Martemyanov, arXiv:0804.3702 [nucl-th].

[17] M. Naruki et al., Phys. Rev. Lett. 96, 092301 (2006) arXiv:nucl-ex/0504016.

[18] K. Ozawa et al. [E325 Collaboration], Phys. Rev. Lett. 86, 5019 (2001) arXiv:nucl-ex/0011013.

[19] D. P. Weygand, C. Djalali, R. Nasseripour and M. Wood [CLAS Collaboration], Int. J. Mod. Phys. A 22, 380 (2007).

[20] R. Arnaldi et al. [NA60 Collaboration], Phys. Rev. Lett. 96, 162302 (2006) arXiv:nucl-ex/0605007.

[21] G. Agakishiev et al. [HADES Collaboration], 
arXiv:0711.4281 [nucl-ex].

[22] G. Agakichiev et al. [HADES Collaboration], Phys. Rev. Lett. 98, 052302 (2007) arXiv:nucl-ex/0608031.

[23] V. Koch and C. Song, Phys. Rev. C 54, 1903 (1996) arXiv:nucl-th/9606028.

[24] C. Ernst, S. A. Bass, M. Belkacem, H. Stoecker and W. Greiner, Phys. Rev. C 58, 447 (1998) arXiv:nucl-th/9712069.

[25] W. Cassing and E. L. Bratkovskaya, Phys. Rept. 308, 65 (1999).

[26] W. Cassing, E. L. Bratkovskaya and S. Juchem, Nucl. Phys. A 674, 249 (2000) arXiv:nucl-th/0001024.

[27] E. L. Bratkovskaya and W. Cassing, arXiv:0712.0635 [nucl-th].

[28] D. Schumacher, S. Vogel and M. Bleicher, Acta Phys. Hung. A 27, 451 (2006) arXiv:nucl-th/0608041.

[29] M. D. Cozma, C. Fuchs, E. Santini and A. Fassler, Phys. Lett. B 640, 170 (2006) arXiv:nucl-th/0601059.

[30] M. Thomere, C. Hartnack, G. Wolf and J. Aichelin, Phys. Rev. C 75, 064902 (2007) arXiv:nucl-th/0702004.

[31] R. Shyam and U. Mosel, Phys. Rev. C 67, 065202 (2003) arXiv:hep-ph/0303035.

[32] L. P. Kaptari and B. Kampfer, Nucl. Phys. A 764, 338 (2006) arXiv:nucl-th/0504072.

[33] I. Augustin, H. H. Gutbrod, D. Kramer, K. Langanke and H. Stocker, arXiv:0804.0177 [hep-ph].

[34] G. S. F. Stephans, J. Phys. G 32, S447 (2006)
arXiv:nucl-ex/0607030

[35] S. A. Bass et al., Prog. Part. Nucl. Phys. 41, 255 (1998) [Prog. Part. Nucl. Phys. 41, 225 (1998)] arXiv:nucl-th/9803035.

[36] M. Bleicher et al., J. Phys. G 25, 1859 (1999) arXiv:hep-ph/9909407.

[37] H. Petersen, M. Bleicher, S. A. Bass and H. Stocker, arXiv:0805.0567 [hep-ph].

[38] L. A. Winckelmann, H. Sorge, H. Stoecker and W. Greiner, Phys. Rev. C 51, 9 (1995).

[39] M. Bleicher and J. Aichelin, Phys. Lett. B 530, 81 (2002) arXiv:hep-ph/0201123.

[40] M. Bleicher, Nucl. Phys. A 715, 85 (2003) arXiv:hep-ph/0212378.

[41] M. Bleicher and H. Stoecker, J. Phys. G 30, S111 (2004) arXiv:hep-ph/0312278.

[42] S. Vogel and M. Bleicher, Rom. Rep. Phys. 58, 63 (2006).

[43] S. Vogel and M. Bleicher, Phys. Rev. C 74, 014902 (2006) arXiv:nucl-th/0509105.

[44] E. L. Bratkovskaya et al., Phys. Rev. C 69, 054907 (2004) arXiv:nucl-th/0402026.

[45] C. Sturm et al. [KAOS Collaboration], Phys. Rev. Lett. 86, 39 (2001) arXiv:nucl-ex/0011001.

[46] All processes where the number of in-going $\rho$ mesons equals the number of outgoing $\rho$ mesons have been discarded from the analysis as they provide only a trivial off-set. 


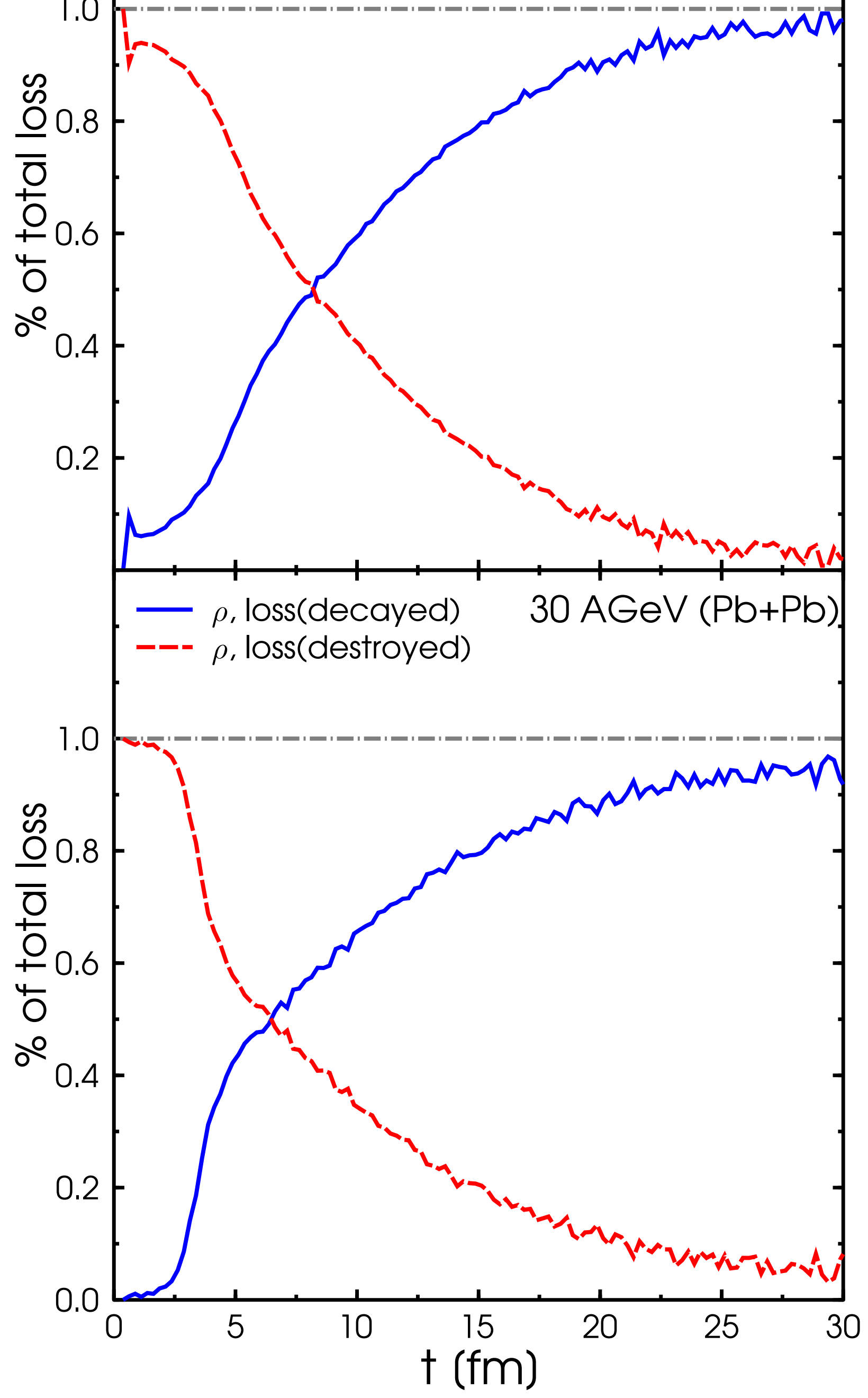

